\title{
Delimitação geográfica da IG do mel de melato de bracatinga do Planalto Sul
}

\section{Brasileiro}

\author{
Geographical delimitation of the IG honeydew honey of bracatinga from the South Brazilian
}

Plateau

Delimitación geográfica del área IG miel de mielada de bracatinga en la Meseta del Sur de Brasil

Recebido: 27/11/2021 | Revisado: 03/12/2021 | Aceito: 06/12/2021 | Publicado: 16/12/2021

Everton Vieira

ORCID: https://orcid.org/0000-0002-0655-5811 Empresa de Pesquisa e Extensão Agropecuária de Santa Catarina, Brasil E-mail: evertonvieira@epagri.sc.gov.br

Denilson Dortzbach

ORCID: https://orcid.org/0000-0002-9470-1072

Empresa de Pesquisa e Extensão Agropecuária de Santa Catarina, Brasil E-mail: denilson@epagri.sc.gov

Kleber Trabaquini

ORCID: https://orcid.org/0000-0003-4902-4735 Empresa de Pesquisa e Extensão Agropecuária de Santa Catarina, Brasil

E-mail: klebertrabaquini@epagri.sc.gov.br

Valci Francisco Vieira

ORCID: https://orcid.org/0000-0003-0554-101X Empresa de Pesquisa e Extensão Agropecuária de Santa Catarina, Brasil E-mail: valci@epagri.sc.gov.br

Fabio Martinho Zambonim

ORCID: https://orcid.org/0000-0001-5046-3589 Empresa de Pesquisa e Extensão Agropecuária de Santa Catarina, Brasil E-mail: zambonim@epagri.sc.gov.br

Arcângelo Loss

ORCID: https://orcid.org/0000-0002-3005-6158 Universidade Federal de Santa Catarina, Brasil E-mail: arcangelo.loss@ufsc.com.br

Paulo Henrique Silva Câmara

ORCID: https://orcid.org/0000-0002-2964-7866

Universidade Federal de Santa Catarina, Brasil E-mail: hpaulo253@gmail.com

\begin{abstract}
Resumo
Os registros de produtos com indicação geográfica (IG) são produzidos em regiões que possuem diferenciais, tais como o local e forma de produção, solos, clima e colheita. Este trabalho objetivou delimitar a área geográfica da IG do Planalto Sul Brasileiro (PSB) de produção de mel de melato de bracatinga através da sobreposição de critérios fitogeográficos, climáticos, geológicos, fisiográficos e produtivos. Foi realizada a delimitação geográfica da área, que teve como base o cruzamento de mapas (político, ortobacias, climático, cobertura e uso das terras), modelo digital de terreno e a localização dos produtores de mel. A estruturação da IG do PSB para o mel de melato de bracantinga busca, além do registro de IG, a participação efetiva dos atores na cadeia produtiva, na tomada de decisão, na viabilização de políticas públicas territoriais e na construção de acordos coletivos. A delimitação geográfica permitiu definir a área de produção que permite a obtenção de produto de qualidades específicas. A área da delimitação geográfica da IG mel de melato de bracatinga do PSB para Santa Catarina é de 43.339,5 km2, correspondendo a 45,3\% do território; no Paraná a área da IG é de 8.500,6 km2, correspondente a 8,9\% do território; e no Rio Grande do Sul é de 7.147,0 km2, cerca de 7,5\% do território. Mais estudos devem ser desenvolvidos na região do PSB visando a influência do meio geográfico sobre o produto Mel de Melato da Bracatinga, assim como sobre as propriedades qualitativas desse mel para demonstrar que esse produto tem características únicas.
\end{abstract}

Palavras-chave: Mimosa scabrella; Denominação de origem; Desenvolvimento sustentável; Floresta Ombrófila Mista; Produto diferenciado.

\footnotetext{
Abstract

The records of products with a geographical indication (GI) are produced in regions that have differences, such as the place and form of production, soil, climate and harvest. This work aimed to delimit the geographical area of GI of Brazilian Southern Plateau (BSP) of honeydew honey bracatinga production through the superposition of
} 
phytogeographic, climatic, geological, physiographic and productive criteria. The geographical delimitation of the area was carried out, based on the crossing of maps (political, orthobasins, climate, land cover and use), digital terrain model, and the location of honey producers. The structuring of GI the PSB for bracatinga honeydew honey seeks, in addition to the GI registration, the effective participation of actors in the production chain, in decision-making, in the feasibility of territorial public policies and in the construction of collective agreements. The geographic delimitation made it possible to define the production area that allows obtaining a product of specific qualities. The area of geographic delimitation of the GI honeydew honey bracatinga from the PSB to Santa Catarina is 43,339.5 km2, corresponding to $45.3 \%$ of the territory; in Paraná the area of the GI is $8,500.6 \mathrm{~km} 2$, corresponding to $8.9 \%$ of the territory; and in Rio Grande do Sul, it's 7,147.0 km2, approximately 7.5\% of the territory. More studies must be carried out in the PSB region aiming at the influence of the geographical environment on honeydew honey bracatinga, as well as on the qualitative properties of this honey to demonstrate that this product has unique characteristics.

Keywords: Mimosa scabrella; Designation of origin; Sustainable development; Mixed Ombrophilous Forest; Differentiated product.

\section{Resumen}

Los registros de productos con indicación geográfica (IG) se elaboran en regiones que presentan diferencias, como el lugar y forma de producción, suelo, clima y cosecha. Este trabajo tuvo como objetivo delimitar el área geográfica de la IG de la Meseta del Sur de Brasil (MSB) de producción de miel de mielada de bracatinga (MMB) mediante la superposición de criterios fitogeográficos, climáticos, geológicos, fisiográficos y productivos. Se realizó la delimitación geográfica del área, a partir del cruce de mapas (político, ortocuencas, clima, cobertura y uso del suelo), modelo digital del terreno y ubicación de productores de MMB. La estructuración de la IG del MSB para la MMB busca, además del registro de IG, la participación efectiva de los actores en la cadena productiva, en la toma de decisiones, en la viabilidad de las políticas públicas territoriales y en la construcción de convenios colectivos. La delimitación geográfica permitió definir la zona de producción que permite obtener un producto de calidades específicas. El área de delimitación geográfica de la IG de MMB de MSB para Santa Catarina es de 43.339,5 km2, correspondiente al 45,3\% del territorio; en Paraná el área es de 8.500,6 km2, correspondiente al 8,9\% del territorio; y en Rio Grande do Sul tiene 7.147,0 km2, aproximadamente el 7,5\% del territorio. Es necesario realizar más estudios en la región de MSB que apunten a la influencia del entorno geográfico en la MMB, así como a las propiedades cualitativas de esta miel para demostrar que este producto tiene características únicas.

Palabras clave: Mimosa scabrella; Denominación de origen; Desenvolvimiento sustentable; Bosque ombrófilo mixto; Producto diferenciado.

\section{Introdução}

O Planalto Sul Brasileiro é a porção meridional do planalto brasileiro estendendo-se pelos estados do Paraná, Santa Catarina e Rio Grande do Sul, e tem na sua constituição geológica rochas efusivas resultantes de erupções vulcânicas, que resultaram em camadas basálticas sobrepostas ao arenito e às rochas cristalinas. Os terrenos são mais elevados próximos ao Oceano Atlântico, alcançando altitudes acima de mil metros, tendo a Serra do Mar e a Serra Geral como uma barreira montanhosa com relevos variados que perdem altitude em direção ao interior (Kern, 1991).

É sobre essa base geológica e geomorfológica que se organizam as formações vegetais do Planalto Sul brasileiro, destacando a Floresta Ombrófila Mista (FOM) que se alterna com os campos cobertos de gramíneas, os chamados campos de cima da serra. É nesta floresta, intermeada com as araucárias que encontramos a bracatinga (Mimosa scabrella) agrupada sob forma de capões de mato ou se estendem sobre as vertentes dos vales de pequenos rios e arroios sob a forma de florestas galerias (Santa Catarina, 1986).

Nesse ambiente, surge o mel de melato da bracatinga, um produto originário do Planalto Sul Brasileiro, nos domínios da Floresta Ombrófila Mista abrangendo grande parte do planalto de Santa Catarina (SC) e adjacências no Rio Grande do Sul (RS) e Paraná (PR) (Dortzbach et al., 2020a).

Obtido a partir da coleta por Apis mellifera das excreções de cochonilhas colonizadoras de caules da árvore bracatinga, o mel de melato da bracatinga é diferenciado quanto as suas propriedades físico-químicas (Campos et al., 2003). O mel de melato passa por dois processos enzimáticos (das cochonilhas e das abelhas) obtendo uma qualidade maior por não cristalizar como o mel floral, isso aliado a uma cor escura típica (Orth \& Martins, 2003; Azevedo, 2017; Azevedo et al., 2021; 
A composição química do mel de melato está diretamente relacionada com os fatores geográficos de sua região de origem (Dortzbach et al., 2020a) e dos aspectos relacionados ao seu processamento, manuseio e armazenamento (MarqueleOliveira et al., 2017).

A qualidade única do produto, em consonância com o planejamento estratégico nacional do setor apícola, que almeja maior participação do Brasil no mercado internacional, fez com que associações de apicultores da região sul do Brasil, tendo como proponente a FAASC (Federação das Associações de Apicultores e Meliponicultores de Santa Catarina) e com o apoio de instituições públicas de pesquisa e extensão rural, como a Epagri em SC, as Ematers do RS e do PR, universidades (UFSC Universidade Federal de Santa Catarina) e Sebrae, se organizaram para encaminhar a documentação necessária para obtenção do registro de Identificação Geográfica (IG) na modalidade Denominação de Origem (DO) do Mel de Melato da Bracatinga do Planalto Sul Brasileiro (Dortzbach et al., 2020a).

A delimitação da área geográfica é uma das etapas fundamentais no processo de reconhecimento de uma IG, conforme Instrução Normativa - IN 95/18 do Instituto Nacional de Propriedade Industrial (INPI) (BRASIL, 2019). A delimitação designa o espaço no qual se realiza a produção e/ou transformação do produto. Neste sentido, a delimitação deve ser realizada e respaldada por argumentos técnicos, como resultados de pesquisas e estudos abordando, principalmente, o levantamento histórico e o estudo de fatores naturais (clima, solo, relevo, vegetação, paisagem, etc.).

A caracterização dos elementos físicos comprova as especificidades observadas no Planalto Sul Brasileiro e serve de subsídio para a solicitação da DO junto ao INPI. O manejo adequado feito pelo apicultor é fundamental para a obtenção do produto mel de melato sem a interferência de mel floral, garantindo assim um produto de qualidade e que atinja as exigências do mercado para se adequar as especificações que constam no regulamento de uso. O mel de melato da bracatinga possui características peculiares, pois é resultado de um conjunto de fatores bióticos e abióticos, com destaque para a relação entre a abelha Apis melíferae a cochonilha do gênero Tachardiella (Dortzbach et al., 2020a).

Neste sentido, o objetivo do presente estudo foi o de realizar a delimitação da área geográfica da IG do Planalto Sul Brasileiro, que é a porção meridional do planalto brasileiro de produção de mel de melato de bracatinga através da sobreposição de critérios fitogeográficos, climáticos, geológicos, fisiográficos e produtivos.

\section{Desenvolvimento}

O presente trabalho foi realizado utilizando-se dados qualitativos e quantitativos, conforme métodos descritos em Pereira et al. (2018). O trabalho foi realizado inicialmente através de pesquisa bibliográfica, desenvolvida através de conteúdos publicados em artigos científicos, teses, dissertações e livros. A busca por termos específicos (mel de melato, bracatinga, meio geográfico, fatores climáticos, fisiografia) tomou como base as plataformas do Scielo, Scopus e Web of Science.

Baseadas nas informações bibliográficas foi realizada a delimitação geográfica da área, que teve como base o cruzamento do Mapa Político de Santa Catarina na escala 1:500.000 (Santa Catarina, 2013); Mapa político do Paraná (PARANÁ, 2015); Base cartográfica do estado do Rio Grande do Sul, escala 1:25.000 (Rio Grande do Sul, 2018); Base Cartográfica da hidrografia Continua do Brasil ao Milionésimo (IBGE, 2014); Mapa das Ortobacias do Brasil (MMA, 2003); modelo digital de elevação (MDE) NASA Resolução de 30 metros (SRTM, 2000); Mapa Climático (Alvares et al., 2013); Monitoramento da cobertura e uso da terra do Brasil IBGE, 2014-2016); Base cartográfica publicada na escala 1:1.000.000, compilado a partir das cartas do Projeto RADAMBRASIL, com escala original de 1:250.000 (IBGE, 2018); e localização dos produtores, através das coordenadas geográficas (FAASC, 2021). Essas bases de informações foram cruzadas no sofware ArcGis 10.4. Com a elaboração do mapa foi possível identificar a participação dos municípios localizados dentro da área geográfica da IG para o mel de melato da bracatinga. 


\section{Resultados e Discussão}

\subsection{Variáveis utilizadas para a delimitação da área}

\section{Área de ocorrência natural da bracatinga}

A bracatinga (Mimosa scabrella Bentham) é uma espécie arbórea, da família Leguminosae: Mimosoideae, característica do Planalto Sul Brasileiro e exclusiva da vegetação secundária da Floresta Ombrófila Mista (Floresta de Araucária), nas formações Montana e Alto-Montana (Carvalho, 2002; IBGE, 2012) (Figura 1). Segundo Klein \& Hatschbach (1962), a distribuição natural geográfica da bracatinga ocorria no primeiro e segundo planalto paranaenses, em praticamente todo o planalto do estado de Santa Catarina, e em parte do Rio Grande do Sul.

$\mathrm{Na}$ vegetação secundária de clareiras naturais ou antrópicas da FOM, a bracatinga apresenta destacada agressividade. Não raro, nessas circunstâncias estabelecem-se, naturalmente, em forma de agrupamentos quase puros. Como elemento exclusivo no extrato superior, em virtude das copas densas, cinzentas e bem formadas, os bracatingais contribuem decididamente na fitofisionomia regional (Carvalho, 1981; Klein et al.; 1978; Bartoszeck, 2000).

Árvore pioneira, típica das regiões mais frias do Sul do Brasil, apresenta características como heliofilia, rusticidade e rápido crescimento em alta densidade (Klein et al., 1981). Nas formações primárias não perturbadas dos pinhais (FOM), principalmente nos de submata densa e bem desenvolvida, a bracatinga é pouco abundante e com dispersão descontínua (Klein, 1981; Carvalho, 2002).

Figura 1. Mapa Fitogeográfico com a abrangência da Floresta Ombrófila Mista (em verde) na região Sul do Brasil.

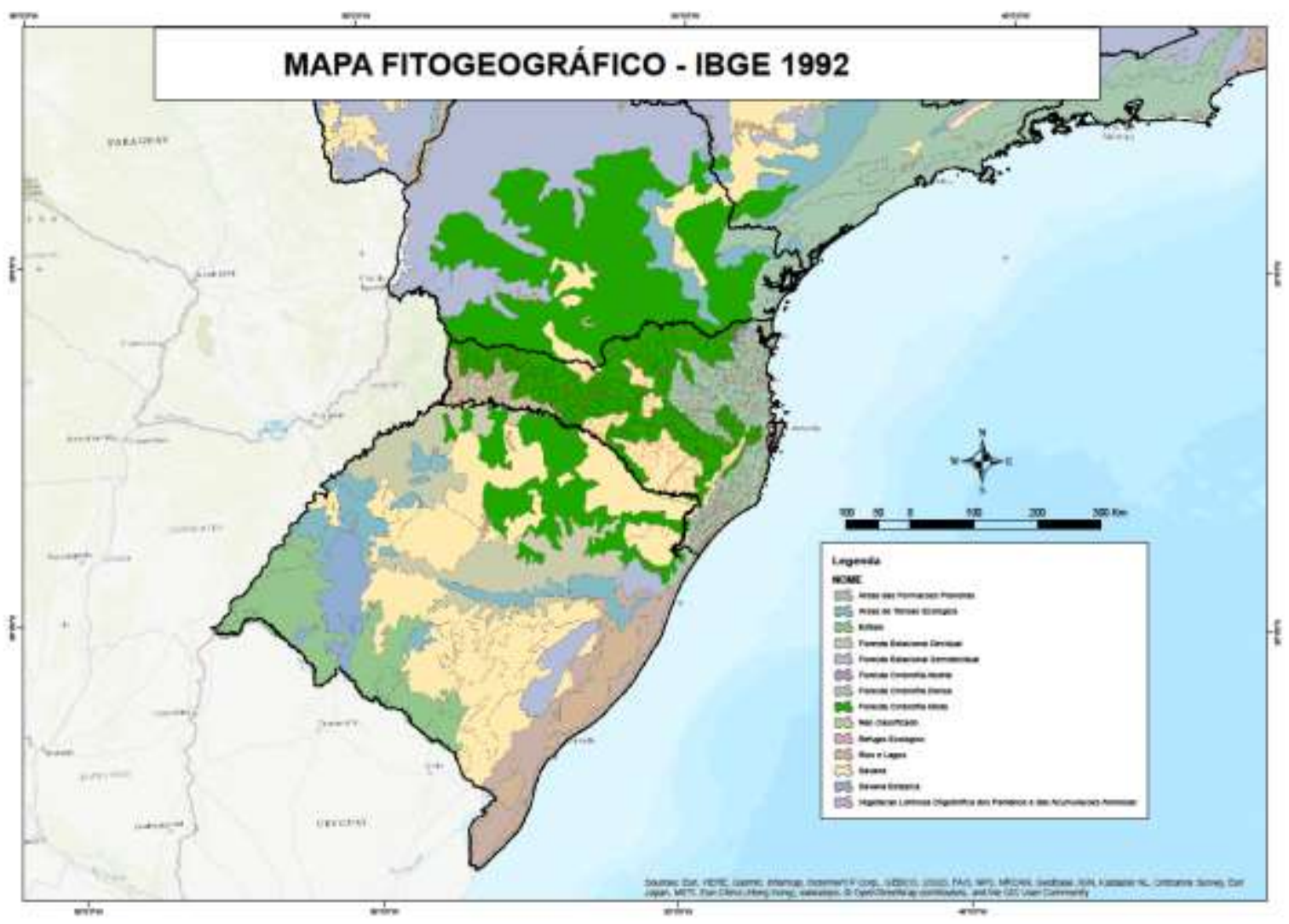

Fonte: IBGE (2012); adaptado de Rangel et al. (1991).

De acordo com Carpanezzi e Laurent (1988), a área mais expressiva e contínua de ocorrência natural da bracatinga situa-se entre as latitudes $23^{\circ} 50^{\prime}$ e $29^{\circ} 40^{\prime} \mathrm{S}$ e compreende terras altas da região Sul e do sul do Estado de São Paulo, na Serra 
do Paranapiacaba. O limite oeste é similar, no Rio Grande do Sul, Santa Catarina e Paraná e situa-se em torno de 5240W. A presença da bracatinga é pouco expressiva no Rio Grande do Sul, comparativamente ao Paraná e Santa Catarina. A bracatinga ocorre, na Região Sul, preferencialmente, nos planaltos, em altitudes acima de 700 metros. Neste estudo foi utilizado o mapa de Rangel et al., (1991), descrito em IBGE (2012).

\section{Variáveis climáticas}

A Mimosa scabrella distribui-se naturalmente na zona subtropical, com invernos rigorosos e umidade constante, sendo resistente a geadas (Hueck, 1972). O clima predominante na região de ocorrência da bracatinga é classificado como Cfb pelo sistema de Koeppen, sendo temperado chuvoso, constantemente úmido, com temperaturas médias do mês mais quente e do mais frio inferiores a $22^{\circ} \mathrm{C}$ e a $18^{\circ} \mathrm{C}$, respectivamente (Figura 2). Carvalho (2002) resume as condições climáticas onde a bracatinga naturalmente se encontra, sendo a precipitação total anual média desde 1.200 mm a 2.300 mm (Paraná e Santa Catarina) com chuvas uniformemente distribuídas na Região Sul. A deficiência hídrica é nula na Região Planalto Sul Brasileiro. Neste estudo foi utilizado o mapa climático Koppen (1948), adaptado por Alvares et al. (2013).

Figura 2. Classificação climática segundo Köppen.

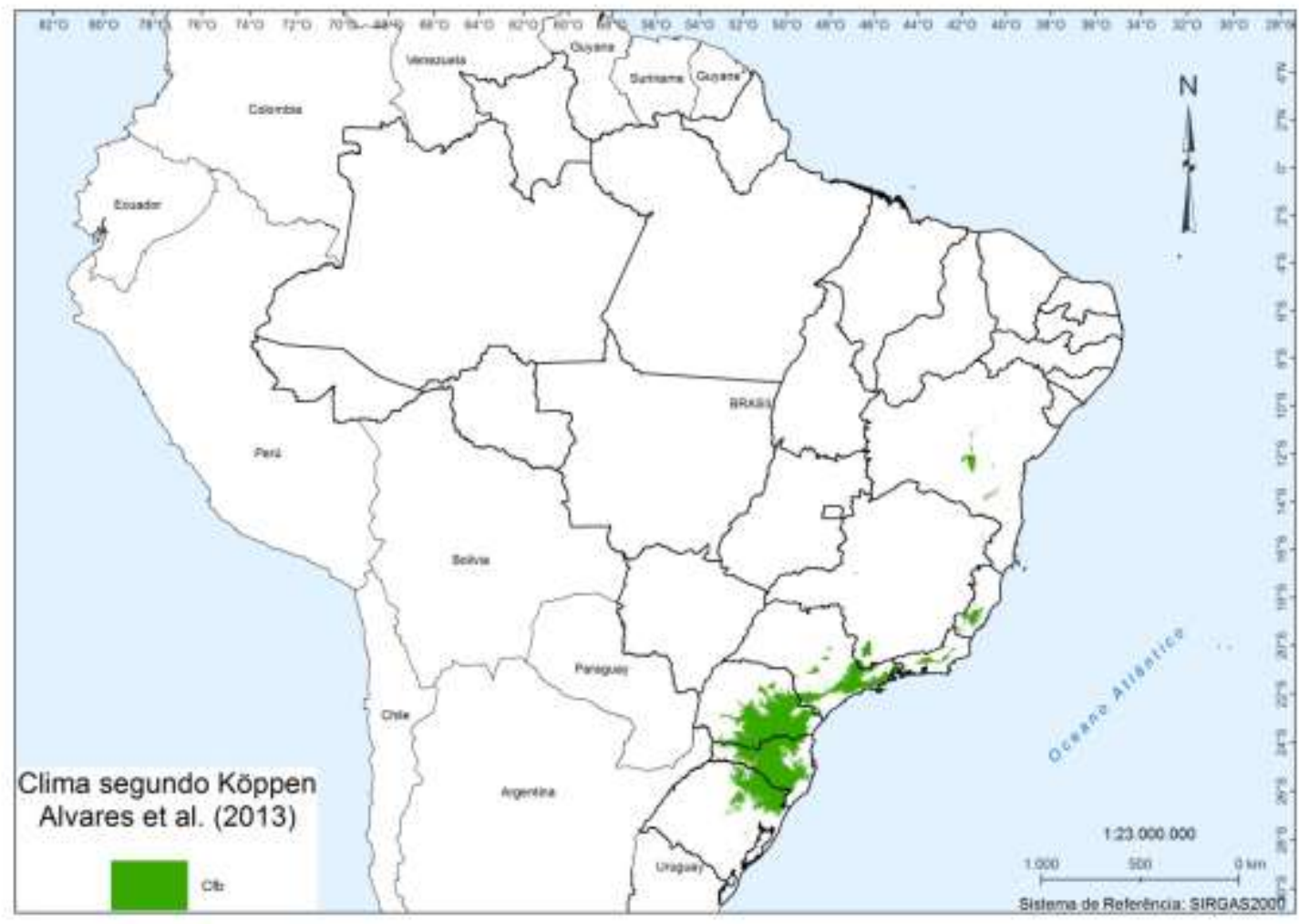

Fonte: Adaptado de Alvares et al. (2013).

\section{Produtores de mel de melato da bracatinga}

A FAASC realizou no ano de 2016 o cadastro dos apicultores catarinenses. Entretanto, este cadastro não diferenciou as produções de mel floral e de mel de melato. Diante disso, foi necessário uma atualização deste cadastro no estado de Santa Catarina, considerando os apicultores que possuem além do mel floral, o mel de melato de bracatinga como fonte de renda.

Assim a FAASC, juntamente com os extensionistas rurais da Epagri, realizaram o levantamento dos apicultores produtores de mel de melato. Para os estados do Paraná e Rio Grande do Sul, como não havia um cadastro georreferenciado 
dos apiários, foi realizado através da Emater em parceria com empresas privadas, o levantamento dos apiários, que serviu de base para o levantamento deste estudo (Figura 3).

Figura 3. Mapa de localização dos produtores de mel de melato de bracatinga.

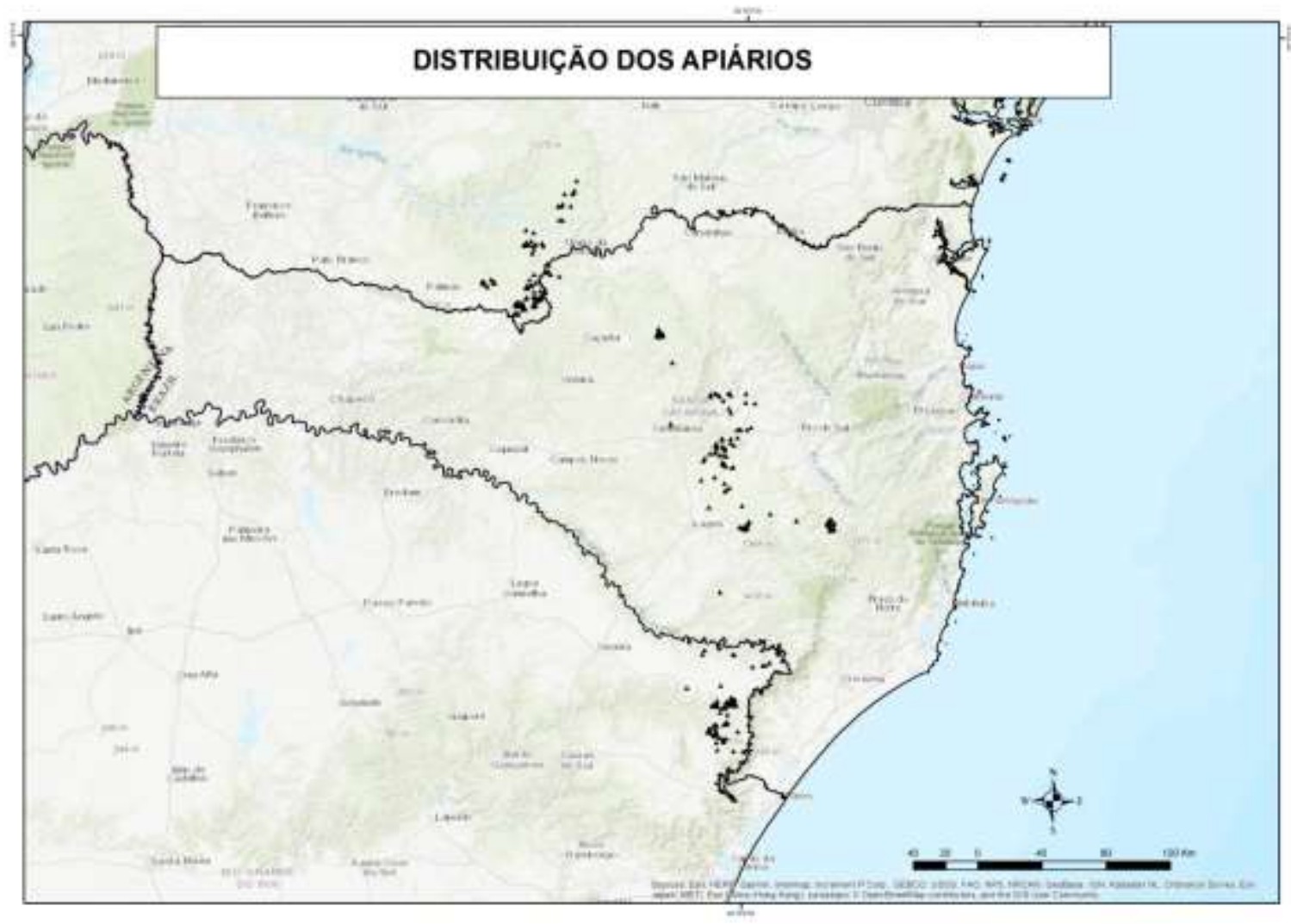

Fonte: Elaborado pelos autores.

\section{Associação entre bracatinga e cochonilhas}

A bracatinga apresenta uma associação com insetos sugadores ao longo dos troncos e galhos conhecidos como cochonilha (Stigmacoccus paranaenses e outras espécies). As cochonilhas, ao se alimentar da seiva da bracatinga, excretam uma grande quantidade de líquido chamado de melato que formam gotículas fixadas num filamento ceroso branco que se comunica com o final do abdômen do inseto. A associação cochonilha-bracatinga ocorre numa área bastante extensa do Planalto Sul Brasileiro onde ocorrem naturalmente bosques de bracatinga. A cochonilha já foi identificada no norte do Rio Grande do Sul (Wolff et al., 2015), em todo o Planalto Catarinense e no Sul do Estado do Paraná. Não está presente em todas as formações de bragatingais e as razões da ausência em vários lugares ainda não é conhecida. Porém, altitudes acima de 700 metros, presença de Floresta Ombrófila Mista com povoamentos de bragatingais e interações climáticas parecem nortear a presença da cochonilha nas bracatingas.

O fenômeno da melação da bracatinga ou presença abundante de gotículas de melato ocorre apenas a cada dois anos, ou seja, é um fenômeno bienal. Porém, a cochonilha está sempre presente, em diferentes estágios de desenvolvimento, na bracatinga. Somente na fase de cistos (ninfas grandes), ela produz copiosamente o melato ao longo dos troncos e galhos da bracatinga (Martins, 2005; Martins-Mansani et al., 2021). Neste período, que coincide com o período de verão/outono dos anos pares, as abelhas coletam o melato e produzem e armazenam o mel de melato de bracatinga nas colmeias. 


\section{Uso da terra}

O Bioma Mata Atlântica é um dos ecossistemas mais degradados do mundo. O estado de Santa Catarina, por exemplo, possuía no ano de 2012, segundo SOS Mata Atlântica (2014), apenas 23,23 \% da cobertura original. Estudos realizados pela Fundação do Meio Ambiente de Santa Catarina concluídos em 2009 (Fatma, 2014), apontam para uma área total de 41,5 \%, coberta de florestas em estagio médio, avançado e/ou primário e segundo Vibrans et al. (2011), o estado ainda apresenta cerca de $36 \%$ da sua cobertura original.

Nas áreas do Planalto Sul Brasileiro, a maior extensão da área ainda coberta por florestas é representada por fragmentos de Floresta Ombrófila Mista, que em muitos locais foi substituída por outros usos do solo (principalmente agricultura e pastagens), ou foram descaracterizadas pela extração de espécies arbóreas de interesse econômico ou ainda foram abandonadas e entraram em processo de sucessão secundária. Dessa forma, essas florestas atualmente estão caracterizadas por mosaicos vegetacionais compostos pelos mais diversos estádios de regeneração (Vibrans et al., 2011).

Esta substituição da vegetação natural causou a diminuição da ocorrência da bracatinga em algumas áreas, principalmente devido a utilização dessas áreas com agricultura e pecuária. Além da diminuição das áreas de bracatinga, a expansão agropecuária também constitui uma barreira para a dispersão da cochonilha, que é fundamental para a produção do mel de melato de bracatinga.

Mesmo em áreas de ocorrência natural da bracatinga, o uso da terra atual define os locais com potencial ou não para a ocorrência do fenômeno e, consequentemente, para a produção de mel de melato de bracatinga. Neste sentido, utilizou-se como material base para a delimitação da área geográfica o mapa de uso e ocupação de solo do IBGE (2014/2016), observado na Figura 4.

Figura 4. Mapa de Uso e Cobertura de Solo com destaque em amarelo das áreas com uso agrícola intensivo.

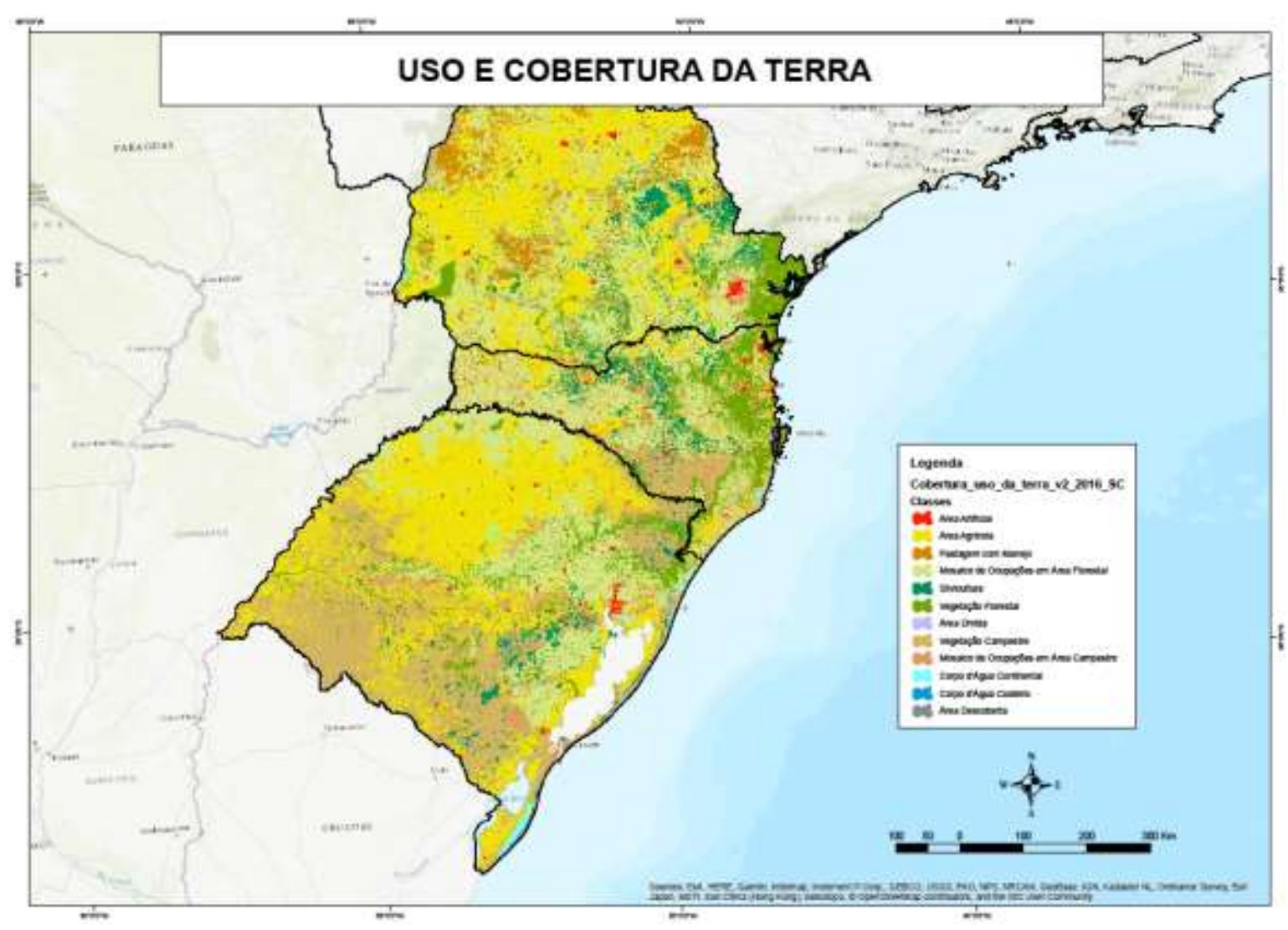

Fonte: IBGE (2014/2016). 


\section{Caracterização química da origem, identidade e tipicidade do mel de melato de bracatinga}

Foram considerados os resultados obtidos a partir da coleta de 34 amostras distintas de mel de melato de bracatinga (Mimosa scabrella Benth.) em locais em que o fenômeno é reconhecido, distribuídos nos estados de Santa Catarina, sul do Paraná e norte do Rio Grande do Sul. As amostras foram submetidas à avaliação dos parâmetros físico-químicos de umidade, acidez total, atividade enzimática (diastase), teor de 5-hidroximetilfurfural, condutividade elétrica, cor, açucares (glicose, frutose, sacarose), pH e os oligoelementos chumbo, antimônio, lântanio, cério, praseodimio, neodimio, samário, európio, gadolinio, térbio, disprósio, hólmio, érbio, túlio, itérbio, lutécio, irídio, platina, ouro, tório, magnésio, alumínio, vanádio, cromo, manganês, ferro, cobalto, cobre, zinco, arsénio, selênio, rubídio, índio, telúrio, césio, bário, titânio, chumbo, urânio, potássio, sódio, cálcio. As respostas analíticas foram submetidas à análise estatística com aplicação do método de análise de componentes principais (PCA, do inglês "principal componente analysis"), o que não permitiu diferenciar as amostras para nenhum dos parâmetros avaliados, concluindo-se que as distintas amostras coletadas, nas diferentes regiões dos três estados do sul do Brasil apresentam características físico-química similares, podendo ser classificadas como o mesmo produto, segundo os parâmetros avaliados. Ainda, a PCA também foi aplicada com o intuito de verificar a distinção entre amostras de mel de melato de bracatinga e dados de méis florais publicados pelo Grupo de Pesquisa em Antioxidantes Naturais (UFSC), considerando os dados de padrões de identidade e qualidade, açúcares e minerais majoritários ( $\mathrm{K}, \mathrm{Mg}, \mathrm{Ca}, \mathrm{Na}$ e $\mathrm{Mn})$. Os resultados demonstraram que as amostras de mel de melato de bracatinga se separam das amostras de méis florais, sendo que as amostras de mel de melato de bracatinga apresentaram escores positivos em relação a esta componente. Tendo em vista que as variáveis de maior peso foram $\mathrm{K}$, condutividade elétrica, $\mathrm{Mg}$ e acidez livre, as amostras de mel de melato de bracatinga se diferenciaram dos méis florais por apresentarem maiores teores de $\mathrm{K}$ e $\mathrm{Mg}$, além de maior condutividade elétrica e acidez (Bergamo et al., 2018a, b; Seraglio et al., 2019; Seraglio et al., 2020; Azevedo et al., 2021).

\section{Delimitação da IG}

Em suma, a FOM no Sul do Brasil, ocorre quase que exclusivamente no planalto sul brasileiro e em clima Cfb. Inserida nesse ecossistema florestal, a bracatinga ocorre natural e preferencialmente em altitudes acima de 700m. É, justamente, neste ambiente que se concentra a atividade econômica da produção de mel de melato da bracatinga, conforme verificado pela espacialização das unidades produtivas (apiários de mel de melato), e que segundo estudos não apresentou diferenças de qualidade em toda a área de abrangência. A partir da análise e do cruzamento dessas informações, e considerando aspectos de uso e ocupação de solo, da divisão politico administrativa municipal e da hidrografia regional foi possível delimitar a área da IG e caracterizar os aspectos ambientais relevantes, que no seu conjunto, garantem a tipicidade e a qualidade do mel de melato da bracatinga do Planalto Sul Brasileiro.

Para a delimitação da área da IG do Mel de Melato da Bracatinga do Planalto Sul Brasileiro foram considerados como variáveis principais a área de ocorrência natural da bracatinga, que está intimamente relacionada com a Floresta Ombrófila Mista e o Clima Cfb. Posteriormente, foi verificada a localização dos apiários de Mel de Melato da Bracatinga em produção. Essas variáveis (FOM, Cfb, Apiários e uso da terra) foram convertidos em planos de informação (PI) no ArcGis para definir o traçado da área delimitada. As análises de mel de melato para toda a região não mostraram diferenças significativas entre as mesmas, o que excluiu este fator para a delimitação da área (Figura 5). 
Figura 5. Mapa climático com os pontos dos produtores de mel de melato de bracatinga.

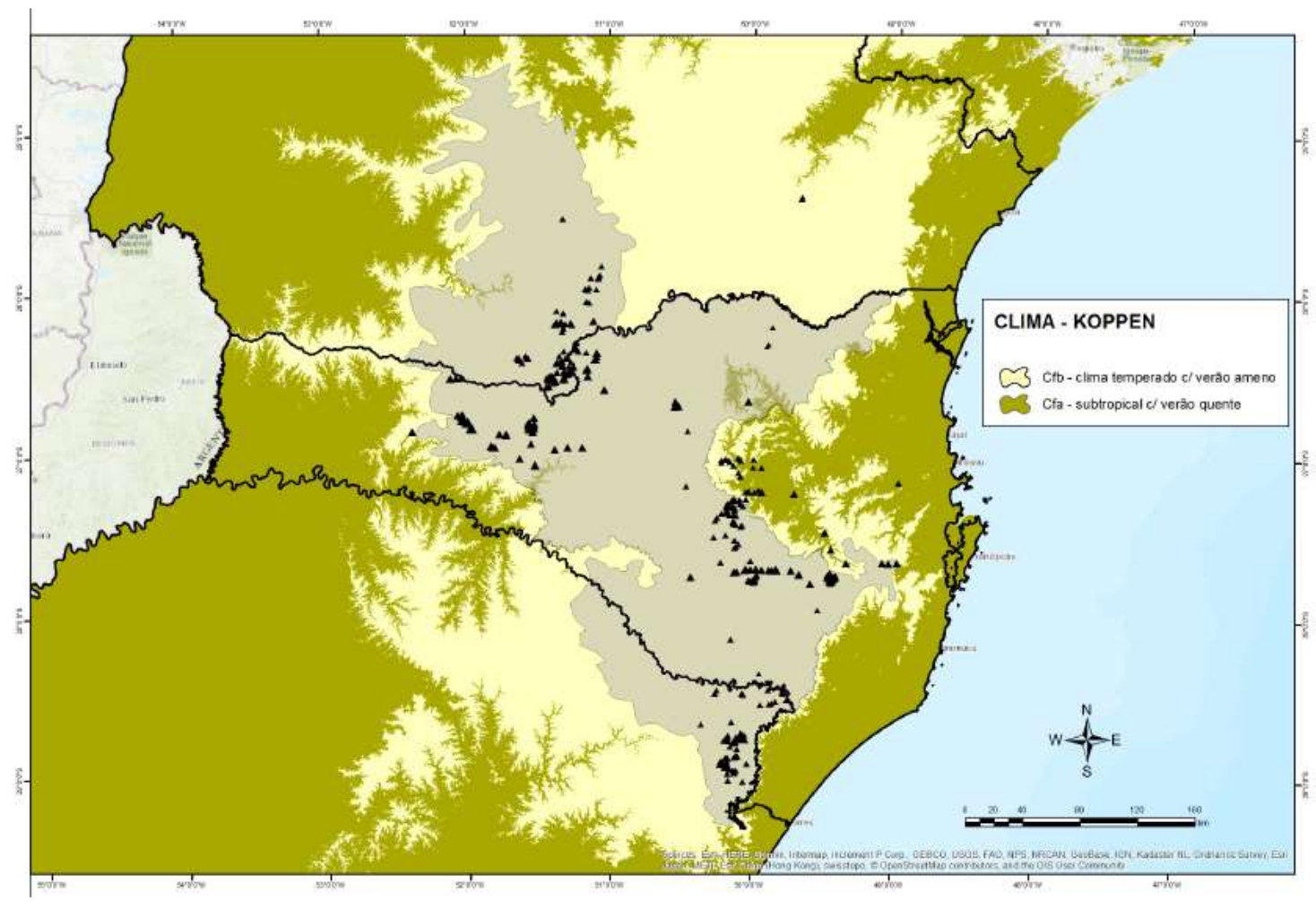

Fonte: Elaborado pelos autores. Os triângulos destacados em preto representam os apiários cadastrados nos três estados avaliados.

É importante destacar que em muitos locais a Floresta Ombrófila Mista ocorre de forma descontínua devido a sua substituição por culturas agrícolas, o que resulta em áreas com pouco ou nenhum bracatingal remanescente. Aliado a isso, a forma de dispersão da cochonilha que se associa a bracatinga é naturalmente muito limitada, o que intensifica a influência do tipo de uso do solo na delimitação da área. A cochonilha, quando associada a bracatinga, apresenta-se imóvel na planta na maior parte do período de seu ciclo de vida. As fêmeas são ápteras e conseguem se deslocar pelo tronco da bracatinga apenas no período do primeiro instar ninfal. Os machos em estágio de pré-pupa são móveis e com pernas bem desenvolvidas e não apresentam tecas alares. Os machos adultos são alados.

Assim, a expansão da agricultura diminui a ocorrência da bracatinga, a dispersão da cochonilha e limita a presença de apicultores, e consequentemente, torna mais restritiva a atividade para os que produzem o mel de melato. Neste sentido, é importante ressaltar que áreas contínuas e ocupadas intensivamente por atividades agropecuárias foram excluídas do território demarcado, usando como base o mapa de uso e ocupação de solo do IBGE (2014/2016).

A próxima etapa foi a definição do traçado da área geográfica, que teve como critério principal a altitude acima de $700 \mathrm{~m}$, definida por muitos autores como a altitude ideal para a ocorrência da bracatinga e a melhor condição para a ocorrência do fenômeno de produção.

Como a altitude possui um limitador de identificação e localização no campo, em algumas áreas, quando possível, utilizou-se a divisa municipal e o divisor de águas (classificação ANA de bacias hidrográficas - OTTO nível 4) para auxiliar na delimitação. Entretanto, em alguns casos, a diversidade dentro dos limites municipais, fez com que a delimitação tivesse necessariamente como limite a cota de $700 \mathrm{~m}$ de altitude. Neste sentido, o traçado segue os limites municipais, divisores de água e a cota altimétrica de 700m, de forma a representar da melhor maneira o território Planalto Sul Brasileiro.

A partir dessas informações espacializadas e sobrepostas e com as variáveis ambientais da área caracterizadas definiuse a delimitação geográfica da Denominação de Origem Mel de Melato da Bracatinga do Planalto Sul Brasileiro. 


\section{Memorial descritivo e Mapa da Delimitação do Território}

Utilizando-se de dados espaciais referenciados ao SIRGAS 2000 com projeção UTM zona 22S, definiu-se a delimitação da área da IG Mel de Melato de Bragatinga do Planalto Sul Brasileiro com uma área de $58.9871 \mathrm{Km}^{2}$. Em Santa Catarina a área delimitada da IG é de 43.339,5 km², correspondendo a 45,3\% do território catarinense; no Paraná a área da IG é de 8.500,6 km², correspondente a 8,9\% do território paranaense e no Rio Grande do Sul é de 7.147,0 km², cerca de 7,5\% do território sul riograndense.

A IG ficou localizada entre os paralelos e meridianos de $25^{\circ} 24^{\prime} 52^{\prime \prime} \mathrm{S}$ e $52^{\circ} 13^{\prime} 24,25^{\prime \prime} \mathrm{W}$ e $29^{\circ} 44^{\prime} 21^{\prime \prime} \mathrm{S}$ e $48^{\circ} 53^{\prime} 76^{\prime \prime} \mathrm{W}$ (Figura 6), abrangendo totalmente e parcialmente os municípios descritos na Tabela 1.

Tabela 1. Lista dos municípios localizados e inseridos total ou parcialmente no território de abrangência da IG de Mel de Melato de Bracatinga.

\begin{tabular}{ll}
\hline Estado & Municípios* \\
\hline & Bom Retiro, Calmon, Capão Alto, Campo Alegre; Campo Belo Do Sul; Campos Novos; Canoinhas; Braço do Trombudo; \\
& Brunópolis; Caçador; Abelardo Luz; Agrolândia; Agua Doce; Alfredo Wagner; Anita Garibaldi; Anitápolis; Arroio Trinta; Bela \\
& Vista Do Toldo; Bocaina Do Sul; Bom Jardim Da Serra; Concordia; Catanduvas; Celso Ramos; Porto União; Correia Pinto; Erval \\
& Velho; Cerro Negro; Curitibanos; Chapadão Do Lageado; Frei Rogerio; Herval Do Oeste; Grão Pará; Ibiam; Ibicare; Fraiburgo; \\
& Irani; Jabora; Jacinto Machado; Irineópolis; Ipira; Abdon Batista; Santa Terezinha; Timbé Do Sul; Treze Tílias; Siderópolis; \\
& Itaiópolis; Monte Carlo; Monte Castelo; Morro Grande; Luzerna; Nova Veneza; Joaçaba; Macieira; Mafra; Major Vieira; \\
& Orleans; Otacílio Costa; Matos Costa; Lacerdópolis; Lages; Mirim Doce; Taió; Tangara; Timbó Grande; Três Barras; Salto \\
& Veloso; Treviso; Vargem Bonita; Videira; Urubici; Urupema; Vargeão; Vargem; Pouso Redondo; Praia Grande; Presidente \\
& Castelo Branco; Rio Do Campo; Petrolândia; Passos Maia; Ouro; Papanduva; Ponte Alta; Ponte Alta Do Norte; Ponte Serrada; \\
& Painel; Palmeira; Peritiba; São José Do Cerrito; São Cristovão Do Sul; São Joaquim; São Bento Do Sul; São Bonifácio; Santa \\
& Cecilia; Santa Rosa De Lima; Lauro Muller; Lebon Regis; Lindoia Do Sul; Rancho Queimado; Rio Fortuna; Rio Negrinho; Rio \\
& Rufino; Pinheiro Preto. \\
\hline PR & Paula Freitas; Coronel Domingos Soares; Mangueirinha; Reserva do Iguaçú; União da Vitória; Pinhão; Cruz Machado; Inácio \\
& Martins; Palmas; General Carneiro; Porto Vitoria \\
\hline \multirow{2}{*}{ RS } & Bom Jesus; Cambará Do Sul; Caraá; Jaquirana; Maquiné; Riozinho; São Francisco De Paula; São José Dos Ausentes; Terra de \\
& Areia, Itati.
\end{tabular}

*Número de municípios abrangidos parcial ou totalmente pelo território da IG, por Estado: SC: 102; PR: 11; RS: 9 municípios.

Fonte: Elaborado pelos autores. 
Figura 6. Mapa da delimitação da IG Mel de Melato da Bracatinga do Planalto Sul Brasileiro.

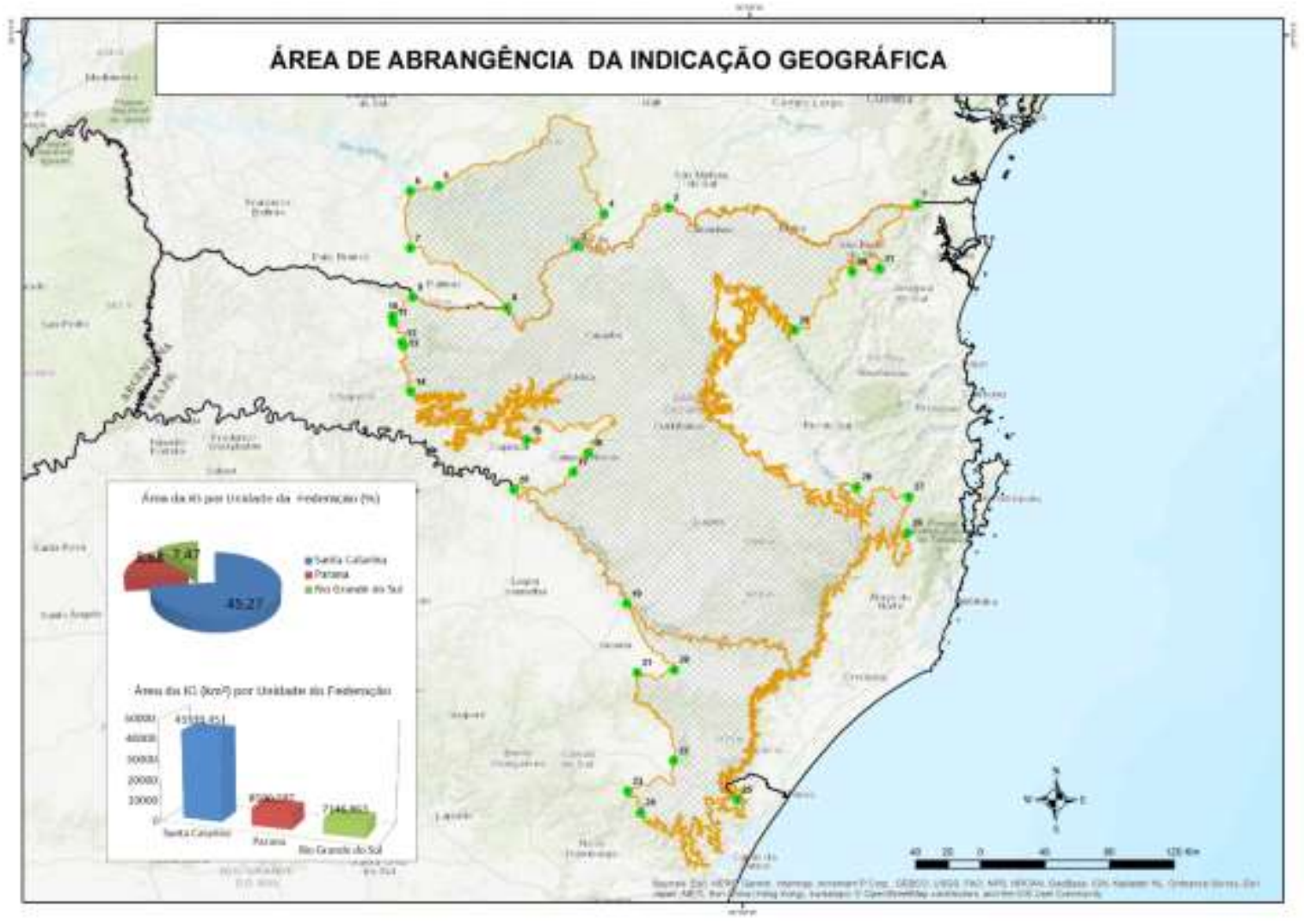

Fonte: Elaborado pelos autores.

Estudos feitos visando a delimitação geográfica da IG da erva-mate e da maçã na região serrana e no Planalto Norte de SC demonstram a importância desses trabalhos, pois as IGs vêm ganhando notória importância no mercado agroindustrial, uma vez que apresenta a competência de diferenciar um produto ou serviço de seus semelhantes, a partir de características naturais (clima, solo, vegetação) e humanas (modo de fazer), tornando, assim, um produto único. Somado a essas características, destaca-se a sua contribuição para a região, que é de grande importância econômica, social e ambiental (Trabaquini et al., 2021; Dortzbach et al., 2020a,b).

\section{Considerações Finais}

A estruturação da Indicação Geográfica do Planalto Sul Brasileiro para o mel de melato de bracantinga busca não apenas a formalização e a conquista de um certificado de registro de uma IG, mas também a participação efetiva dos atores (principalmente apicultores) na cadeia produtiva, na tomada de decisão, na viabilização de políticas públicas territoriais e na construção de acordos coletivos.

A delimitação geográfica permitiu definir a área de produção que permite a obtenção de um produto de qualidades específicas. A área da delimitação geográfica da IG mel de melato de bracatinga do Planalto Sul Brasileiro para Santa Catarina é de 43.339,5 km2, correspondendo a 45,3\% do território catarinense; no Paraná a área da IG é de 8.500,6 km2, correspondente a 8,9 \% do território paranaense e no Rio Grande do Sul é de 7.147,0 km2, cerca de 7,5\% do território sul riograndense.

Mais estudos devem ser desenvolvidos na região do Planalto Sul Brasileiro visando a influência do meio geográfico sobre o produto Mel de Melato da Bracatinga, assim como sobre as propriedades qualitativas desse mel para demonstrar que esse produto tem características únicas. 


\section{Referências}

Alvares, C. A., Stape, J. L., Sentelhas, P. C., Gonçalves, J. L. M. \& Sparovek, G. (2013). Köppen's climate classification map for Brazil. Meteorologische Zeitschrift, 22(6), 711-728.

Azevedo, M. S. (2017). Mel de melato de bracatinga (Mimosa scabrella Bentham) do Planalto Serrano de Santa Catarina: discriminação e potencialidade de alimento funcional. Tese (Doutorado em Ciência dos Alimentos) - Programa de Pós-Graduação em Ciência dos Alimentos, Universidade Federal de Santa Catarina, Florianópolis. 201p.

Azevedo, M. S., Seraglio, S. K. T., Bergamo, G., Rocha, G. O., Valese, A. C., Daguer, H., Miotto, M., Gonzaga, L. V., Fett, R. \& Costa, A. C. O. (2021). Physicochemical properties and biological activities of bracatinga honeydew honey from different geographical locations. Journal of Food Science and Technology-Mysore, 58, 3417-3429.

Bartoszeck, A. C. P. S. (2000). Evolução da relação hipsométrica e da distribuição diamétrica em função dos fatores idade, sítio e densidade inicial em bracatingais da Região Metropolitana de Curitiba. Dissertação (Mestrado em Engenharia Florestal) - Universidade Federal do Paraná, Curitiba. 214f.

Bergamo, G., Seraglio, S. K. T., Gonzaga, L. V., Fett, R. \& Costa, A. C. O. (2018a). Physicochemical characteristics of bracatinga honeydew honey and blossom honey produced in the state of Santa Catarina: An approach to honey differentiation. Food Research International, 116,745-754.

Bergamo, G., Seraglio, S. K. T., Gonzaga, L. V., Fett, R. \& Costa, A. C. O. (2018b). Mineral profile as a potential parameter for verifying the authenticity of bracatinga honeydew honeys. LWT-Food Science and Technology, 97, 390-395.

Campos, G., Della-Modesta, R. C., Silva, T. J. P., Baptista, K. E., Gomides, M. F. \& Godoy, R. L. (2003). Classificação do Mel em Floral ou Mel de Melato. Revista de Ciência e Tecnologia de Alimentos, 23(1), 1-5.

Carpanezzi, A. A. \& Laurent, J. E. (1988). Manual técnico da bracatinga (Mimosa scabrella Benth). Embrapa. CNPF. Curitiba. (Documentos, 20). 70p.

Carvalho, P. E. R. (1981). Composição e crescimento de um povoamento natural de bracatinga (Mimosa scabrella Bentham.). In: Seminário sobre atualidades e perspectivas florestais, 4., 1981, Curitiba PR. Bracatinga uma alternativa para reflorestamento: Anais... Curitiba: Embrapa-UFPRCS, 145-148p. (EmbrapaUFPRCS. Documentos, 5).

Carvalho, P. E. R. (2002). Bracatinga. Colombo: Embrapa/CNPF. 12p. (Circular Técnica 59).

Copé, S.M. (2015). A gênese das paisagens culturais do planalto sul brasileiro. Estudos Avançados. 29(83), $149-171$.

Dortzbach, D.; Machado, L. N., Loss, A. \& Vieira, E. (2020a). Influência do meio geográfico nas características do mel de melato da bracatinga. Research, Society and Development, 09(9), e198997191.

Dortzach, D., Machado, L. N., Loss, A., Vieira, V. F., Ricce, W. S., Pereira, M. G., Queiroz Santos, O. A. \& Netto, A. J. J G. O. (2020b). Delimitação geográfica da área da IG erva-mate do Planalto Norte Catarinense. Research, Society and Development, 9 (10), e5029108769.

Escuredo, O., Míguez, M., Fernández-González, M. \& Seijo, M. C. (2013). Nutritional value and antioxidant activity of honeys produced in a European Atlantic area. Food Chemistry, 138(2-3), 851-856.

FAASC - Federação das Associações de Apicultores e Meliponicultores de SC. Inventário da Apicultura Catarinense. (2017). Florianópolis: FAASC. http://www.faasc.com.br/noticiasid/107.

Fatma - Fundação do Meio Ambiente. Atlas da cobertura vegetal de Santa Catarina. (2014). http://www.fatma.sc.gov.br/conteudo/atlas-da-cobertura-vegetalde-santa-catarina.

Fundação SOS Mata Atlântica \& Instituto Nacional de Pesquisas Espaciais - INPE. Atlas dos Remanescentes Florestais da Mata Atlântica: período 20112012. (2014). http://www.sosma.org.br/link/atlas201112/ATLAS_apresentacao_2011_2012_COLETIVA.pdf.

IBGE - Instituto Brasileiro de Geografia e Estatística. (2009). Manual Técnico de Geomorfologia. Rio de Janeiro: Diretoria de Geociências. 2009. 175p.

IBGE - Instituto Brasileiro de Geografia e Estatística. (2012). Manual Técnico da Vegetação Brasileira. Rio de Janeiro: Diretoria de Geociências. 272p.

IBGE - Instituto Brasileiro de Geografia e Estatística. (2017). Produção da Pecuária Municipal. Rio de Janeiro: Diretoria de Pesquisas. 45, 1-8.

IBGE - Instituto Brasileiro de Geografia e Estatística. (2020). Monitoramento da cobertura e uso da terra do Brasil 2014-2016. Mapas em meio digital Escala 1:1.000.000 - Datum Sirgas 2.000 - projeção policônica. https://www.ibge.gov.br/apps/monitoramento_cobertura_uso_terra/v1

IBGE - Instituto Brasileiro de Geografia e Estatística. (2018). Mapa da Vegetação Brasileira. Banco de dados georeferenciado sobre recursos naturais. Base cartográfica publicada na escala 1:1.000.000, compilado a partir das cartas do Projeto RADAMBRASIL, com escala original de 1:250.000. Http://geoftp.ibge.gov.br/informacoes_ambientais/vegetacao/vetores/escala_250_mil.

IBGE - Instituto Brasileiro de Geografia e Estatística. (2014). Base Cartográfica da hidrografia Continua do Brasil ao Milionésimo.

Http://geoftp.ibge.gov.br/cartas_e_mapas/bases_cartograficas_continuas/bcim/versao2016

Kern, A. A. (1991). Arqueologia Pré-histórica do Rio Grande do Sul. Mercado Aberto.

Klein, R. (1978). Mapa fitogeográfico de Santa Catarina. In: Reitz, P. Flora Ilustrada de Santa Catarina. Itajaí: Herbário Barbosa Rodrigues, p. 1-24.

Klein, R. M. (1981). Aspectos fitossociológicos da Bracatinga (Mimosa scabrella). In: Seminario sobre atualidades e perspectivas florestais, 4., Curitiba PR. Bracatinga uma alternativa para reflorestamento: anais... Curitiba: Embrapa-UFPRCS, p.145-148. (Embrapa-UFPRCS. Documentos, 5). 
Klein, R. M. \& Hatschbach, G. (1962). Fitofisionomia e notas sobre a vegetação para acompanhar a planta fitogeográfica do município de Curitiba e arredores (Paraná). Curitiba. Boletim da Universidade Federal do Paraná, Geografia Física, 4:1-29.

Marquele-Oliveira, F., Carrão, D. B., Souza, R. O., Baptista, N. U., Nascimento, A. P., Torres, E. C., Moreno, G. P., Buszinski, A. F. M., Miguel, F. G., Cuba, G. L., Reis, T. F., Lambertucci, J., Redher, C. \& Berretta, A. A. (2017). Fundamentals of Brazilian Honey Analysis: An Overview. In: Toledo, V.A.A. (ed). Honey Analysis. InTech, 139-170.

Martins, M. (2005). Interação entre Tachardiella sp. (Homoptera) e Mimosa scabrella Benth. (Leguminosae) e a produção de mel de melato por Apis mellifera L. (Hymenoptera). Dissertação. Programa de Pós-Graduação em Recursos Genéticos Vegetais - Florianópolis. 93p.

Martins-Mansani, M.; Faita, M. R.; Orth, A.I. \& Nodari, R.O. (2021). Production of Honeydew by Scale Insects Associated with Bracatinga (Mimosa scabrella Benth) in Serra Catarinense, Southern Brazil. Revista Brasileira de Entomologia, 65, e20200072.

MMA. (2003). Mapa das Ortobacias do Brasil. http://mapas.mma.gov.br/i3geo/datadownload.htm

Orth, A; Martins, M. (2003). Diversidade e abundância de insetos sobre as secreções de Tachardiella sp. (Homoptera: Lacifferidae) em troncos de bracatinga (Mimosa scabrella) no planalto de Santa Catarina. In: Congresso de Ecologia do Brasil, 6., Fortaleza, CE. Anais...Fortaleza: UFCE, p.573-575.

Paraná. Instituto de Terras, Cartografia e Geologia do Paraná (2015). Divisão Político Administrativa do Paraná. Mapa político. http://www.itcg.pr.gov.br/modules/faq/category.php?categoryid=8\# -

Pereira A. S., Shitsuka, D. M., Parreira, F. J. \& Shitsuka, R. (2018). Metodologia da pesquisa científica. UFSM.

Reitz, R., Klein, R. M. \& Reis, A. (1978). Projeto madeira de Santa Catarina. Itajai: SUDESUL: Herbário Barbosa Rodrigues, 320 p.

Rio Grande do Sul. (2018). Base cartográfica do estado do Rio Grande do Sul. Escala 1:25.000 - bcrs25 versão 1.0 - Porto Alegre. http://ww2.fepam.rs.gov.br/bcrs25/.

Santa Catarina. Mapa político (2013). Mapa Político de Santa Catarina (1:500.000): Secretaria de Estado do Planejamento: Diretoria de Geografia e Cartografia.

Srtm. Shuttle Radar Topography Mission. (2000). MDE NASA. Resolução de 30 metros. SRTM 2000. https://www.dlr.de/eoc/en/desktopdefault.aspx/tabid5515/9214_read-17716/

Seraglio, S. K. T., Silva, B., Bergamo, G., Brugnerotto, P., Gonzaga, L. V., Fett, R. \& Costa, A. C. O. (2019). An overview of physicochemical characteristics and health-promoting properties of honeydew honey. Food Research International, 119, 44-66.

Seraglio, S. K. T., Silva, B., Bergamo, G., Brugnerotto, P., Gonzaga, L. V., Fett, R. \& Costa, A. C. O. (2020). Aliphatic organic acids as promising authenticity markers of bracatinga honeydew honey. Food Chemistry, 343, 28449.

Trabaquini, K., Lunardi, N., Vieira, V. F., Dortzach, D., Vieira, E., Ricce, W. S., Loss, A., Blainski, E., Zambonim, F. M. \& Silveira, J. C. (2021). Identificação e espacialização dos pomares de maçã utilizando dados de sensoriamento remoto no estado de Santa Catarina-Brasil. Revista de Ciências Agrárias, 44(2), 114-126

Vibrans, A. C., Sevegnani, L., Uhlmann, A., Schorn, L. A., Sobral, M., Gasper, A. L., Lingner, D. V., Brogni, E., Klemz, G., Godoy, M. B. \& Verdi, M. (2011). Structure of mixed ombrophyllous forests with Araucaria angustifolia (Araucariaceae) under external stress in Southern Brazil. Revista de Biologia Tropical, 59, 1371-1387.

Vieira, A. C. P., Maestrelli, S. R. \&Arcari, S. G. (2014). Cartilha da Indicação de Procedência dos Vales da Uva Goethe. Florianópolis, SC: Epagri. 20p.

Wolff, V. R. S., Witter, S. \& Lisboa, B. B. (2015). Reporte de Stigmacoccus Paranaensis Foldi (Hemiptera, Stigmacoccidae), insecto escama asociado con la producción de miel de mielato en Rio Grande do Sul, Brasil. Insecta Mundi, 0434, 1-7. 